Mahmood ESFANDIARI ${ }^{1}$

Saber SAATI ${ }^{1,2}$

Mehrzad NAVABAKHSH ${ }^{1}$

Kaveh KHALILI-DAMGHANI ${ }^{1}$

\title{
A NOVEL DATA ENVELOPMENT ANALYSIS MODEL WITH COMPLEX NUMBERS. MEASURING THE EFFICIENCY OF ELECTRIC GENERATORS IN STEAM POWER PLANTS
}

The output of a generator in power plant is the electricity, and it consists of two parts, active and reactive power. These quantities are expressed as complex numbers in which the real part is the active power and the imaginary part is the reactive power. Reactive power plays an important role in an electricity network. Ignoring it will exclude a lot of information. With regard to the importance of the generators in power plants, surely, calculating the efficiency of these units is of great importance. Data Envelopment Analysis (DEA) is a nonparametric approach to measure the relative efficiency of Decision-Making Units (DMUs). Since the generators data are complex numbers, thus, if we the use classical DEA models in order to measure the efficiency of the generators in power plants, the reactive power cannot be considered, and the measurement is limited to the real number of electric power. In this paper, a new DEA model with complex numbers is developed in order to assess the performance of the power plant generators.

Keywords: Data Envelopment Analysis, power plant, power plant efficiency, complex numbers

\section{Introduction}

Data Envelopment Analysis (DEA) is a well-known nonparametric approach based on linear programming used to measure the efficiency of a group of independent homogenous Decision-Making Units (DMUs) with multiple inputs and outputs presented

${ }^{1}$ Department of Industrial Engineering, Faculty of Industrial Engineering, South Tehran Branch, Islamic Azad University, Tehran, Iran, e-mail addresses: Mahmood.esfandiari2010@yahoo.com, kkhalili@azad.ac.ir, mnavabakhsh@azad.ac.ir

${ }^{2}$ Department of Mathematics, North Tehran Branch, Islamic Azad University, Tehran, Iran, e-mail address: s_saatim@iau-tnb.ac.ir 
by Charnes et al. [5]. This model is known as CCR model. Since 1978, DEA has had significant progress in various fields such as production, service, healthcare, economy, banking, insurance, agriculture, energy, and military sections. Sengupta [18] propose the stochastic DEA incorporating the stochastic programming which combines the models of data envelopment analysis with the Chance Constraint Programming (CCP). Sengupta [19] first uses fuzzy sets in DEA models and utilized three types of fuzzy statics, i.e., fuzzy mathematical program, fuzzy regression, and fuzzy entropy, in order to show the available solutions with ambiguous data. Cooper et al. [7] apply DEA using interval and ordinal data and utilise a unified IDEA model with weight restrictions. Banker [1] adds statistical elements to the efficiency evaluation techniques of DEA. Portela et al. [16] develop a new DEA model that can be used with negative data and implement this model to bank branches. Wen et al. [20] develop a DEA model with uncertain input and output data.

In terms of data types, all extensions and developments of DEA models have used real number data sets, while there are some practical applications into which complex numbers are involved. For instance, we can consider the power plant industry, mechanic industry or telecommunication industry, etc. In the mechanical industry, for example in oscillators, the total force applied for oscillators is defined as a complex number [8]. In telecommunication systems, information is received and transmitted in the form of electrical signals the data of which have a complex nature [3]. In the same way, in the electric power industry, generators of power-plants convert the mechanical power into electric power. The output data of the generators in power plants (i.e., the electric power) is available as a complex number consisting of two main parts, namely active power (real part) and reactive power (imaginary part).

There are studies in the field of DEA to evaluate the efficiency of power plants. Park and Lesourd [14] examine the efficiencies of 64 steam-fuel power plants in South Korea using DEA and stochastic frontier. Sarika and Or [17] apply DEA to assess the efficiencies of 65 thermal, water, and wind power plants in private and public sectors in Turkey. Liu et al. [10] assess the efficiency of the thermal power plants from 2004 to 2006 using the DEA method. Khalili-Damghani et al. [9] propose a DEA model using interval data and undesirable outputs in order to assess the performance of the combined cycle power plants. $\mathrm{Wu}$ and $\mathrm{Li}$ [21] investigate the efficiency of the Chinese wind power industry from the perspective of capital raise and allocation using the DEA model. Chandel et al. [4] calculate the performance of state-owned thermal power plants in northern India in the period 2009-2014 using DEA. Lyu and Shi [11] present a DEA model to assess the efficiency of different parts of the global renewable energy industry and then to apply their model to Bloomberg New Energy Finance (BNEF) data.

Similar to the above studies, if traditional DEA models are used to measure the efficiency of the power plants with real numbers, the reactive power cannot be measured, and it is thus ignored. Therefore, the measurement is limited to the real number of electric power, i.e., to active power, while reactive power plays an important role in the 
power generation network. The reactive power is applied to manage the voltage level to push the active power through the lines and, if there is no reactive power, there is no possibility of transferring power demanded through the lines and, ultimately, the useful performance is not produced $[2,12]$. Thus, calculating the efficiency of the power plant using classical models of DEA that ignore the imaginary part of the electric power, i.e., the reactive power, does not yield real efficiency.

None of the classical DEA models can deal with complex numbers. Such a technical gap in the field of classical the DEA models leads us to develop a new DEA model with complex numbers. For developing the novel model that uses the concept of the size of virtual input and output, we transform the assumed model into an NLP model, then further transform it into an LP model by interchanging variables. Eventually, the proposed model is used in a real case study in order to measure the efficiency of electric generators in power plants.

The present paper is organized as follows: In Section 2, the theoretical preliminaries to DEA method and complex numbers are stated. In Section 3, a new DEA model considering the complex numbers is introduced. In Section 4, the results of applying the proposed DEA model in a case study of generators of power-plants are presented. Finally, Section 5 provides concluding remarks and future research directions.

\section{Theoretical preliminaries}

\subsection{Classical DEA model}

DEA is a linear programming (LP) method to assess the efficiency of several DMUs with multiple inputs and outputs [5]. Suppose that $n$ DMUs are assessed by a DEA model in such a way that each DMU consumes $m$ inputs to produce $s$ outputs, where $x_{i j}$ represents $i$ th $(i=1, \ldots, n)$ input and $y_{r j}$ represents the $r$ th $(r=1, \ldots, s)$ output of the $j$ th $(j=1, \ldots, n)$ DMU. The CCR model to measure the efficiency score of $\mathrm{DMU}_{p}$ is formulated as follows:

$$
\begin{aligned}
& \min z=\theta \\
& \text { s.t.: } \\
& \sum_{j=1}^{n} \lambda_{j} x_{i j} \leq \theta x_{i p}, \quad i=1, \ldots, m \\
& \sum_{j=1}^{n} \lambda_{j} y_{r j} \geq y_{r p}, \quad r=1, \ldots, s \\
& \lambda_{j} \geq 0, \quad j=1, \ldots, n
\end{aligned}
$$


model (1) is known as the input-oriented envelopment model under constant returns to scale (CRS) assumption. The first constraint compares the size of the inputs of $p$ th unit with the size of the weighted sum of inputs of all units, and the second constraint compares the size of the outputs of $p$ th unit with the size of the weighted sum of outputs of all units. In the real number set, the size of a real number is equal to its value.

\subsection{Complex numbers}

A complex number is defined as $a+i b$, where $a$ and $b$ are real numbers and $i$ is an imaginary number with its square being $-1\left(i^{2}=-1\right)$. In this general form, $a$ and $b$ are called the real and imaginary parts, respectively. The complex number set is represented by $C$. Since the complex number $a+i b$ is represented exclusively by an ordered pair $(a, b)$, the complex numbers have one-to-one correspondence with the points on the plane called a complex plane. Suppose $z=a+i b, z_{1}=a_{1}+i b_{1}$ and $z_{2}=a_{2}+i b_{2}$ are three complex numbers and $k \in \mathbb{R}$, then the following relations hold true [6])

$$
\begin{aligned}
& |z|=\sqrt{a^{2}+b^{2}} \\
& k z=k a+i k b, \quad k \in \mathbb{R} \\
& z_{1}+z_{2}=\left(a_{1}+a_{2}\right)+i\left(b_{1}+b_{2}\right)
\end{aligned}
$$

In the above relation, the sign || represents the length, modulus or the size of a complex number.

\section{The new DEA model with complex numbers}

Consider $n$ DMU and that each DMU consumes $m$ complex inputs $x_{i j}=x_{i j}^{a}+i x_{i j}^{b}$, $i=1, \ldots, m$ to produce $s$ complex outputs $y_{r j}=y_{r j}^{a}+i y_{r j}^{b}, r=1, \ldots, s$, where $x_{i j}^{a}$ and $x_{i j}^{b}$ are called the real and imaginary parts of the inputs, respectively. Also $y_{r j}^{a}$ and $y_{r j}^{b}$ are called the real and imaginary parts of the outputs, respectively. It should be noted that the real and imaginary parts of the inputs and outputs are assumed to be non-negative, and that each DMU has at least one input and output greater than zero. As mentioned earlier, in the constraints of model (1), the size of the combined inputs and outputs of all DMUs is compared to the size of inputs and outputs of $\mathrm{DMU}_{p}$, respectively. By considering this notion and the complex data in model (1), we have: 
$\min \mathrm{z}=\theta$

s.t.:

$$
\begin{aligned}
& \left|\sum_{j=1}^{n} \lambda_{j}\left(x_{i j}^{a}+i x_{i j}^{b}\right)\right| \leq \theta\left|\left(x_{i p}^{a}+i x_{i p}^{b}\right)\right|, \quad i=1, \ldots, m \\
& \left|\sum_{j=1}^{n} \lambda_{j}\left(y_{r j}^{a}+i y_{r j}^{b}\right)\right| \geq\left|y_{r p}^{a}+i y_{r p}^{b}\right|, \quad r=1, \ldots, s \\
& \lambda_{j} \geq 0, \quad j=1, \ldots, n, \quad \theta \text { free }
\end{aligned}
$$

Taking into account the properties of complex numbers presented in relations (2), model (3) is converted into the model (4):

$$
\begin{aligned}
& \min z=\theta \\
& \text { s.t.: } \\
& \left|\sum_{j=1}^{n} \lambda_{j} x_{i j}^{a}+i \sum_{j=1}^{n} \lambda_{j} x_{i j}^{b}\right| \leq \theta\left|\left(x_{i p}^{a}+i x_{i p}^{b}\right)\right|, \quad i=1, \ldots, m \\
& \left|\sum_{j=1}^{n} \lambda_{j} y_{r j}^{a}+i \sum_{j=1}^{n} \lambda_{j} y_{r j}^{b}\right| \geq\left|y_{r p}^{a}+i y_{r p}^{b}\right|, \quad r=1, \ldots, s \\
& \lambda_{j} \geq 0, \quad j=1, \ldots, n, \quad \theta \text { free }
\end{aligned}
$$

Sequentially, according to the definition in (2), the size of a complex number is calculated as the second root of the sum of squares of real and imaginary parts. Due to the comparison structure of the constraints of model (4) and for simplification, we can use the second power of the size of a complex number. model (4) is converted as follows:

$\min z=\theta$

s.t.:

$\sum_{j=1}^{n} \lambda_{j}^{2}\left(\left(x_{i j}^{a}\right)^{2}+\left(x_{i j}^{b}\right)^{2}\right)+2 \sum_{j=1}^{n-1} \sum_{h=j+1}^{n} \lambda_{j} \lambda_{h}\left(x_{i j}^{a} x_{i h}^{a}+x_{i j}^{b} x_{i h}^{b}\right) \leq \theta\left(\left(x_{i p}^{a}\right)^{2}+\left(x_{i p}^{b}\right)^{2}\right), i=1, \ldots, m$

$\sum_{j=1}^{n} \lambda_{j}^{2}\left(\left(y_{r j}^{a}\right)^{2}+\left(y_{r j}^{b}\right)^{2}\right)+2 \sum_{j=1}^{n-1} \sum_{h=j+1}^{n} \lambda_{j} \lambda_{h}\left(y_{r j}^{a} y_{r h}^{a}+y_{r j}^{b} y_{r h}^{b}\right) \geq\left(\left(y_{r p}^{a}\right)^{2}+\left(y_{r p}^{b}\right)^{2}\right), r=1, \ldots, s$

$\lambda_{j}, \lambda_{h} \geq 0, \quad j=1, \ldots, n, \quad h=2, \ldots, n, \quad \theta$ free 
Model (5) is a non-linear programming (NLP) problem and it is difficult to achieve a global optimal solution. We use McCormick's [13] proposed transformation and consider the variables substitution $\lambda_{j j}=\lambda_{j}^{2}$ and $\lambda_{j h}=\lambda_{j} \lambda_{h}$. By replacing these changes in model (5), the following LP model is obtained:

$\min z=\theta$

s.t.:

$\sum_{j=1}^{n} \lambda_{j j}\left(\left(x_{i j}^{a}\right)^{2}+\left(x_{i j}^{b}\right)^{2}\right)+2 \sum_{j=1}^{n-1} \sum_{h=j+1}^{n} \lambda_{j h}\left(x_{i j}^{a} x_{i h}^{a}+x_{i j}^{b} x_{i h}^{b}\right) \leq \theta\left(\left(x_{i p}^{a}\right)^{2}+\left(x_{i p}^{b}\right)^{2}\right), \quad i=1, \ldots, m$

$\sum_{j=1}^{n} \lambda_{j j}\left(\left(y_{r j}^{a}\right)^{2}+\left(y_{r j}^{b}\right)^{2}\right)+2 \sum_{j=1}^{n-1} \sum_{h=j+1}^{n} \lambda_{j h}\left(y_{r j}^{a} y_{r h}^{a}+y_{r j}^{b} y_{r h}^{b}\right) \geq\left(\left(y_{r p}^{a}\right)^{2}+\left(y_{r p}^{b}\right)^{2}\right), \quad r=1, \ldots, s$

$\lambda_{j j}, \lambda_{j h} \geq 0, \quad j=1, \ldots, n, \quad h=2, \ldots, n, \quad \theta$ free

The above LP problem is an input-oriented DEA model with complex numbers under CRS assumption, and it is developed to evaluate the efficiency of DMUs incorporating complex inputs and outputs.

Lemma 1. Model (6) is feasible.

Proof. Consider $\theta=1, \quad \lambda_{j j}=0, \lambda_{j h}=0, \forall j, h, j \neq p, h \neq p$, and $\lambda_{p p}=1$. This solution satisfies all constraints of model (6). So, it is a feasible solution of model (6).

Theorem 1. The optimal value of model (6) is between 0 and 1 .

\section{Proof.}

A. Assume that $\theta^{*}<0$. Therefore, the right-hand side of the first set of constraints in model (6) will be negative. On the other hand of this constraint, we know that

$$
\sum_{j=1}^{n} \lambda_{j j}\left(\left(x_{i j}^{a}\right)^{2}+\left(x_{i j}^{b}\right)^{2}\right)+2 \sum_{j=1}^{n-1} \sum_{h=j+1}^{n} \lambda_{j h}\left(x_{i j}^{a} x_{i h}^{a}+x_{i j}^{b} x_{i h}^{b}\right) \geq 0
$$

and this will lead to conflict since a negative term is set to be greater than or equal to a positive term. Hence, $\theta^{*} \geq 0$.

B. Assume that $\theta^{*}=0$. So, all $\lambda_{j j}$ and $\lambda_{j h}$ in the first set of constraints in model (6) should be equal to zero. Therefore, the left-hand side of the second set of constraints in model (6) gets zero value and, consequently, the right-hand side of the second constraint 
becomes zero, and this will lead to a conflict. Since there is at least one output with a positive value. Therefore, $\theta^{*} \neq 0$.

C. Due to Lemma 1, since model (6) is feasible with $\theta=1$, and this model is a minimization problem, it is concluded that $\theta^{*} \leq \theta$. Hence $\theta^{*} \leq 1$. This completes the proof.

\section{Application of new proposed DEA model in power plants}

\subsection{Real case study of power plants}

We have applied the proposed model to a real case study in order to measure the efficiency of steam power plants generators. A thermal power plant (steam power plant) is a power station in which the electric generator is steam driven. The generator consists of two main parts of the rotor (rotating part) and stator (static part). In the rotating section, a rotating magnetic field is generated using excitation current and voltage, and in the stator coil voltage or electricity is produced. In fact, the generator converts mechanical energy created by the turbine into electrical energy. The most important part of a thermal power plant is the generator. The greatest variation in the design of steamelectric power plants is due to the different fuel sources. Worldwide, most electric power is produced by thermal-electric power plants, which produce about $86 \%$ of all electricity generation.

It should be mentioned that the efficiency of generators of the power plants as main parts of power plants has not been evaluated by DEA models, and in the present study, we will focus on measuring the performance of the generators of thermal power plants due to their undeniable roles in power generation. The complex nature of the power generation in plants makes the proposed model in this study appropriate for the assessment. In the present study, the input and output data are available as real and complex numbers. The output of the generators, i.e., the electric power, consists of complex numbers with two parts: the active power $\left(y^{a}\right)$ is considered the real part and reactive power $\left(y^{b}\right)$ is considered the imaginary part of a complex number in the calculations. In other words, the output power of the generator is represented as $y=y^{a}+i y^{b}$. To this end, the annual data of 14 generators of the steam power plants (as DMUs) in Iran for the year 2015 were selected. The power plants are supported by Iran Electricity Management, Distribution, Transfer, and Production Company (known as Tavanir Company). Table 1 represents data of each power plant in 2015.

As we mentioned earlier, the mechanical energy, excitation voltages, and excitation current are considered as inputs of generators, and the output of generators is electrical power that appears in both active power and reactive power and is sent to the consumption network. Figure 1 presents the schematic view of a generator in a steam power plant as a DMU. 
Table 1. Inputs and outputs data of the power plants

\begin{tabular}{|c|l|c|c|c|c|c|}
\hline DMU & \multicolumn{1}{|c|}{ Power plant } & $x_{1}$ & $x_{2}$ & $x_{3}$ & $y_{b}$ & $y_{a}$ \\
\hline 1 & Shahid Salimi Neka & 3100 & 280 & 440 & 50 & 400 \\
\hline 2 & Beeston Kermanshah & 2800 & 222 & 289 & 32 & 270.4 \\
\hline 3 & Besat Tehran & 980 & 170 & 65.4 & 10 & 60 \\
\hline 4 & Shahid Firuzi Tehran & 870 & 180 & 11.2 & 2 & 10 \\
\hline 5 & Shazand & 2900 & 230 & 284 & 27 & 270 \\
\hline 6 & Esfahan & 1280 & 237 & 154 & 17 & 140 \\
\hline 7 & East Azerbaijan (Sahand) & 2800 & 250 & 277 & 29 & 260 \\
\hline 8 & Iranshahr & 1125 & 198 & 57 & 7 & 51.9 \\
\hline 9 & Hormozgan & 2980 & 240 & 263 & 30 & 250 \\
\hline 10 & Shahid Beheshti Loshan & 789 & 159 & 94.6 & 8.6 & 84.9 \\
\hline 11 & Mofatteh Hamedan & 1670 & 279 & 193.2 & 20 & 175 \\
\hline 12 & Ramin Ahvaz & 2710 & 230 & 279 & 23 & 260.1 \\
\hline 13 & Shahid Rajaee Ghazvin & 2600 & 250 & 225 & 22 & 210 \\
\hline 14 & Zarand Kerman & 680 & 180 & 23.1 & 9.1 & 19 \\
\hline
\end{tabular}

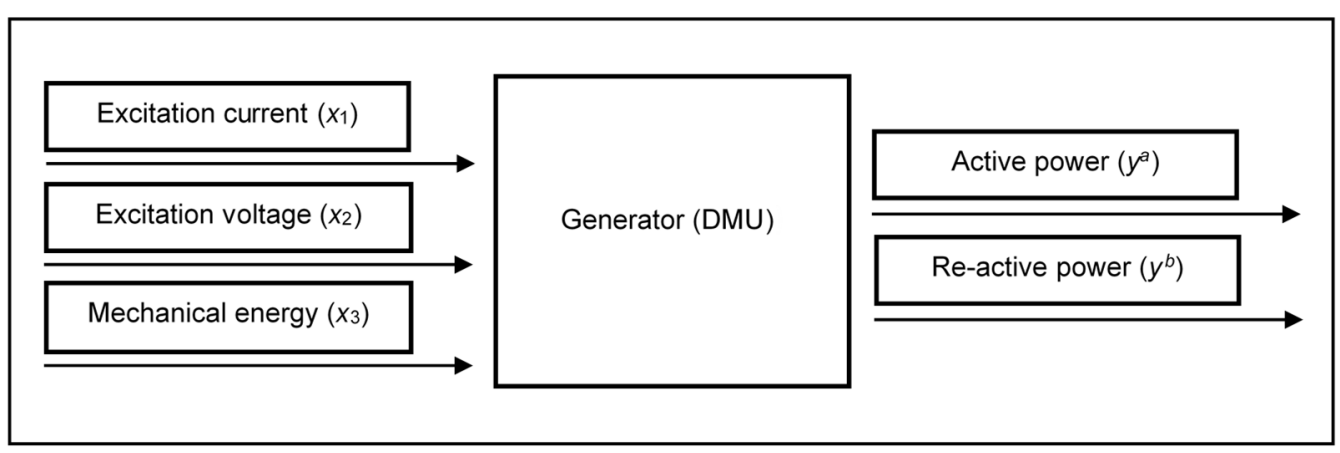

Fig. 1. Schematic view of a generator as a DMU

The inputs and outputs of the generators are defined as follows:

The excitation current and voltage $x_{1}, x_{2}$ - to create a magnetic field, the rotor coil of the generator should be fed by a current and voltage. In other words, the machine rotor is powered by a high-power electronic converter. These inputs have real nature.

Mechanical power $x_{3}$ - the power that is generated by the kinetic energy of steam turbines enters into the generator and has a real nature.

Electric power $(y)$ - the voltage or power that is generated in the stator section of the generator and is emitted by the generator. This output data have complex nature.

Active power $y_{r j}^{a}-$ it is a part of the electric power that is sent through the transmission lines and can carry out a certain job. For instance, it rotates electric motors or lights up lights. It is measured in megawatt. This power is defined as the real part of a complex number. 
Reactive power $y_{r j}^{b}$ - this power is a part of the electric power, it is sent with active power to the consumer networks, and its performance is not seen with the eye (for example, does not light the lamp). It manages the voltage level to send active power through the lines. Its unit of measurement is megavar. This power is defined as the imaginary part of a complex number.

\subsection{Results and discussion}

The proposed DEA model (6) was coded using the GAMS software in which the output of the model shows the efficiency scores of 14 DMUs. Table 2 shows the efficiency scores of generators of steam power plants measured with the proposed DEA model (6).

Table 2. Efficiency scores of the generators based on the DEA model (6)

\begin{tabular}{|r|l|c|}
\hline DMU & \multicolumn{1}{|c|}{ Power plant } & Efficiency score \\
\hline 1 & Shahid Salimi Neka & 1.000 \\
\hline 2 & Beeston Kermanshah & 0.990 \\
\hline 3 & Besat Tehran & 0.944 \\
\hline 4 & Shahid Firuzi Tehran & 0.905 \\
\hline 5 & Shazand & 1.000 \\
\hline 6 & Esfahan & 0.966 \\
\hline 7 & East Azerbaijan (Sahand) & 0.980 \\
\hline 8 & Iranshahr & 0.925 \\
\hline 9 & Hormozgan & 1.000 \\
\hline 10 & Shahid Beheshti Loshan & 0.936 \\
\hline 11 & Mofatteh Hamedan & 0.952 \\
\hline 12 & Ramin Ahvaz & 0.973 \\
\hline 13 & Shahid Rajaee Ghazvin & 0.961 \\
\hline 14 & Zarand Kerman & 0.915 \\
\hline
\end{tabular}

It can be concluded from Table 2 that $\mathrm{DMU}_{1}, \mathrm{DMU}_{5}$, and $\mathrm{DMU}_{9}$ are considered efficient DMUs. $\mathrm{DMU}_{4}$ enjoys the lowest rate of efficiency. These results were not very far from one's mind because high and low efficiencies of the generators depend on the design of the generators or their technology. As an example, consider DMU $\mathrm{D}_{1}$ (Shahid Salimi Power Plant in Neka) located on the coast of Caspian Sea. This power plant has a large share in producing the electricity required by the national network of Iran and it is considered to be the strategic power plants of the country and also one of the most important national assets. The generators in this unit are designed in such a way as to have good reactions to the severe hits of the active power and reactive power. $\mathrm{DMU}_{4}$ (Shahid Firouzi Power Plant), located in Tehran (Tarasht district), is one of the oldest 
power plants in Iran in which the steam units were manufactured and installed in the electricity generation cycle in the late 1950s. Considering the results of applying the research model, this power plant had the lowest efficiency value. In order to check the effect of including reactive power in the assessment of the efficiency of the generators in power plants, an analysis has been conducted. First, suppose that the imaginary part of the power is ignored in all power plants. Then, in the case study, we measure the efficiency of using just the real part of the electric power. Here, model (1) is used in order to calculate the efficiency of power plant generators. Table 3 shows the comparative results of the classical DEA model, the proposed DEA model, and traditional efficiency measurement.

Table 3. Efficiencies calculated based on various models compared with the traditional efficiency

\begin{tabular}{|r|l|c|c|c|}
\hline DMU & \multicolumn{1}{|c|}{ Power plant } & Model (1) & Model (6) & $\begin{array}{c}\text { Traditional } \\
\text { efficiency }\end{array}$ \\
\hline 1 & Shahid Salimi Neka & 1 & 1 & 0.980 \\
\hline 2 & Beeston Kermanshah & 0.981 & 0.990 & 0.965 \\
\hline 3 & Besat Tehran & 0.956 & 0.944 & 0.915 \\
\hline 4 & Shahid Firuzi Tehran & 0.949 & 0.905 & 0.880 \\
\hline 5 & Shazand & 0.993 & 1 & 0.970 \\
\hline 6 & Esfahan & 0.988 & 0.966 & 0.920 \\
\hline 7 & East Azerbaijan (Sahand) & 0.990 & 0.980 & 0.960 \\
\hline 8 & Iranshahr & 0.960 & 0.925 & 0.900 \\
\hline 9 & Hormozgan & 0.997 & 1 & 0.970 \\
\hline 10 & Shahid Beheshti Loshan & 0.939 & 0.936 & 0.910 \\
\hline 11 & Mofatteh Hamedan & 0.960 & 0.952 & 0.930 \\
\hline 12 & Ramin Ahvaz & 0.984 & 0.973 & 0.950 \\
\hline 13 & Shahid Rajaee Ghazvin & 0.977 & 0.961 & 0.940 \\
\hline 14 & Zarand Kerman & 0.865 & 0.915 & 0.890 \\
\hline
\end{tabular}

It should be mentioned that the traditional efficiency of power generators is calculated on the basis of the amount of energy produced (electricity) compared to the amount of energy consumed. The results of model (1), model (6), and traditional evaluation of efficiency are presented in Table 3. In order to investigate the correlation between the efficiencies, the Pearson correlation coefficient is utilised. The correlation coefficient between the obtained efficiency from the proposed DEA model and traditional efficiency is 0.979 , and the correlation coefficient between the obtained efficiency from the classical DEA model and traditional efficiency is 0.756 . These results show that there is a higher correlation between the efficiencies obtained from the proposed DEA model and the traditional assessment of the efficiency. It can be concluded that measuring the efficiency of generators in power plants considering imaginary part of outputs provides more reliable results which are aligned with the results of the traditional evaluation of efficiency. 


\section{Concluding remarks and future research directions}

Assessing the efficiency of the generators in power plants can be of great importance for various managerial purposes. Since the output data of a generator is made up of a complex number, the classical DEA models cannot provide reliable results in calculating the efficiency of the generator. Here, none of the classical DEA models can deal with complex numbers. If we use the classical DEA models which use real numbers in order to measure the efficiency of the generators of the power plants, the reactive power cannot be measured, and it is actually ignored. In this case, the measurement is limited to the real number of electric power, i.e., active power.

In this paper, we initially address the existing DEA models to develop a DEA model using the complex numbers. Then, we elaborate the complex numbers and eventually propose a new DEA model considering complex numbers. With respect to the case study, we develop the input-oriented DEA model under CRS condition in order to assess the efficiency of DMUs using complex numbers. In order to check the applicability of the proposed model, we apply the model to the data set from 14 generators of steam power plants in Iran. The proposed models are coded in GAMS software. The results of the model reveal that the proposed DEA model given in this study is capable to measure the efficiency score of DMUs considering complex data. To clarify the main purpose of the research and its advantage over previous studies, we calculate the efficiency of the power plants, regardless of the imaginary part of the parameters (as real numbers) using the classical DEA model. The efficiency scores achieved by the proposed model and the classical DEA model are compared with the traditional efficiency measurement. The higher correlation coefficient between the results of the proposed model and the traditional efficiency shows a similarity of the results of these two efficiencies. So, incorporating the imaginary part of the parameters and using the proposed DEA model with complex numbers can be considered as a more acceptable method for evaluating the efficiency of steam power plants generators. One of the benefits of the proposed model is that it can simultaneously investigate the effect of the real and imaginary part of the data on efficiency. This is done by considering the size of the data combination rather than the size of the data in the model. Whenever we want to use the data size in the model, although it will be very simple, it may lead to incorrect results.

The proposed model in this study can be extended for network DEA, dynamic DEA, and Malmquist models with complex numbers. Applying the present research approach to evaluate processes with complex negative, fuzzy, undesirable, and uncertain data can be interesting areas of study.

\section{Acknowledgements}

The authors would like to thank the Iran Electricity Management, Distribution, Transfer, and Production Company (known as Tavanir Company) for collecting data and consultation. 


\section{References}

[1] BANKer R.D., Maximum likelihood, consistency and data envelopment analysis. A statistical foundation, Manage. Sci., 1993, 39 (10), 1265-1273.

[2] Bhola J.C., Jyotishi P., Reactive power compensation in $132 \times 33 \mathrm{kV}$ grid of Narsinghpur Area, Int. J. Comp. Eng. Res., 2016, 6 (6), 6-15.

[3] Carlson A., Crilly P., Rutledge J., Communication Systems, McGraw-Hill Series in Electrical and Computer Engineering, 2001.

[4] Chandel R., Singh H., Kumar R., Performance evaluation of state-owned thermal power plants in northern India using DEA, J. Global Energy Iss., 2017, 40 (6), 380-399.

[5] Charnes A., COOPER W.W., RHOdes E., Measuring the efficiency of decision-making units, Eur. J. Oper. Res., 1978, 2, 429-444.

[6] Churchill R.V., Brown J.W., Verhey R.F., Complex Variables and Applications, McGraw-Hill, New York 1974.

[7] COOPER W.W., PARK K.S., YU G.I., Models for dealing with imprecise data in DEA, Manage. Sci., 1999, 45, 597-607.

[8] Fowles G., Cassiday G., Analytical Mechanics, Cengage Learning, Inc., CA. United States, 2004.

[9] Khalili-Damghani K., TAVANA M., HaJI-SAAmi E., A data envelopment analysis model with interval data and undesirable output for combined cycle power plant performance assessment, Exp. Syst. Appl., 2015, 42 (2), 760-773.

[10] LIU C.H., LiN S.J., LEWIS C., Evaluation of thermal power plant operational performance in Taiwan by data envelopment analysis, Energy Pol., 2010, 32 (2), 1049-1058.

[11] LYU X., SHI A., Research on the renewable energy industry financing efficiency assessment and mode selection, Sust., MDPI, Open Access J., 2018, 10 (1), 1-13.

[12] Miller T.E., Reactive Power Control in Electric Systems, Wiley, New York 1983.

[13] MCCormick G.P., Computability of global solutions to factorable nonconvex programs. Part I. Convex underestimating problems, Math. Program., 1976, 10, 147-175.

[14] PARK S.U., LESOURD J.B., The efficiency of conventional fuel power plants in South Korea. A comparison of parametric and non-parametric approaches, Int. J. Prod. Econ., 2000, 63 (1), 59-65.

[15] Pearson K., Mathematical contributions to the theory of evolution. Part III. Regression, heredity, and panmixia, Phil. Trans. R Soc. Lond., Series A, 1896, 187, 253-318.

[16] Portela M., Thanassoulis E., Simpson G., Negative data in DEA. A directional distance approach applied to bank branches, J. Oper. Res. Soc., 2004, 55 (10), 1111-1121.

[17] SARIKA K., OR A., Efficiency assessment of Turkish power plants using data envelopment analysis, Energy, 2007, 32 (8), 1484-1499.

[18] SenguPTA J.K., A fuzzy system approach in data envelopment analysis, Comp. Math. Appl., 1992, 24, 259-266.

[19] Sengupta J.K., Stochastic programming, Int. J. Syst. Sci., 1982, 7, 822-835.

[20] Wen M., Guo L., Kang R., Yang Y., Data envelopment analysis with uncertain inputs and outputs, Appl. Math., 2014, 2, 1-7.

[21] Wu L., Li H., Analysis of the development of the wind power industry in China from the perspective of the financial support, Energy, Sust., Soc., 2017, 7 (37), 7-37.

Received 2 July 2017 Accepted 4 January 2020 